\title{
Tolerance and outcome of hypofractionated post-mastectomy radiotherapy among elderly breast cancer patients in a specialized center in Nigeria
}

\author{
Usman Malami Aliyu ${ }^{1}$, Awosan Kehinde ${ }^{2}$ \\ ${ }^{1}$ Departmet of Radiotherapy and Oncology, Usmanu Danfodiyo University/Teaching Hospital Sokoto, Sokoto, Nigeria; ${ }^{2}$ Department of Community \\ Health, Usmanu Danfodiyo University/Teaching Hospital Sokoto, Sokoto, Nigeria \\ Contributions: (I) Conception and design: UM Aliyu; (II) Administrative support: A Kehinde; (III) Provision of study materials or patients: UM Aliyu; \\ (IV) Collection and assembly of data: UM Aliyu; (V) Data analysis and interpretation: A Kehinde; (VI) Manuscript writing: All authors; (VII) Final \\ approval of manuscript: All authors. \\ Correspondence to: Usman Malami Aliyu. Department of Radiotherapy and Oncology, Usmanu Danfodiyo University/Teaching Hospital Sokoto, \\ Sokoto, Nigeria. Email: usmangandi@yahoo.com.
}

Background: The incidence of breast cancer rises dramatically with age, and numerous studies have
reported the benefits of post mastectomy radiotherapy in both young and elderly breast cancer patients.
The limited numbers of radiotherapy machines and the conventional fractionation schedule which are of
long duration make access to radiotherapy more difficult especially among the elderly in Nigeria. Hypo
fractionated radiotherapy offers an alternative option (with a shorter duration and comparable side effects
and outcomes) to conventional radiotherapy as reported in western literatures. Such studies are grossly
lacking in a sub-Saharan African country like Nigeria, hence the need for this study.
Methods: In this retrospective study, the records of 83 elderly patients with stages II and III breast cancer
that were treated with radiotherapy in the Radiotherapy and Oncology Department of UDUTH, Sokoto,
Nigeria, from January 2015 to February 2019 were evaluated. Radiotherapy was given at a dose of 45 Gy in
18 fractions of 2.5 Gy to the chest wall and the regional lymph nodes over a period of 3.5 weeks. The end
point was freedom from any grade 3 or higher toxicities and disease free survival at 2 years.
Results: Majority, 67 ( $80.7 \%$ of the 83 patients had invasive ductal carcinoma which were not otherwise
specific. About two-thirds of the patients ( $69.9 \%$ ) had the disease located in the right breast. After a 24 month
follow up, there were no grade 3 or 4 toxicities. Forty-two (50.6\%) and 16 (19.2\%) patients had grade 2 skin
and nausea/vomiting toxicities respectively. Eighty (96.4\%) were disease free at 24 months, 3 (3.6\%) patients
had local recurrence, while 1 (1.2\%) had distant metastasis to the lungs. The two years overall survival rate
was $90 \%$.

Conclusions: Hypofractionated radiotherapy in elderly breast cancer patients proved to be comparable to conventional radiotherapy in terms of toxicities and outcome in our center.

Keywords: Advanced age; breast cancer patients; hypofractionated radiotherapy; Nigeria; outcome

Submitted Mar 04, 2020. Accepted for publication Oct 18, 2020.

doi: $10.21037 /$ tcr-20-1335

View this article at: http://dx.doi.org/10.21037/tcr-20-1335

\section{Introduction}

Cancer incidence and mortality are rapidly growing worldwide. The reasons are complex but it reflects both aging and growth of population, as well as changes in the prevalence and distribution of the main risk factors for cancer, several of which are associated with socioeconomic development (1). Worldwide, there were about 2.1 million newly diagnosed breast cancer cases in 2018, accounting 
for almost 1 in 4 cancer cases among women. Breast cancer is the most diagnosed and also the leading cause of cancer deaths in over 100 countries worldwide (2).

The incidence of breast cancer rises dramatically with age, which is the single most important risk factor for developing cancer (1). As a consequence of the combination of increasing life expectancy and early breast cancer screening programs, we are witnessing a rapid rise in both developed and developing countries of breast cancer in the elderly. For example, in the USA over $40 \%$ of patients diagnosed with early breast cancer are aged 65 years or older (3). In the UK, of the 48,417 cases of breast cancer, $45 \%$ (approximately 21,000 ) of the patients were aged $\geq 65$ years (3). Definitions of 'elderly' or 'older' patients vary between 65 years and older in North America to 70 years or older in Europe $(4,5)$. By 2030, the proportion of older adults (65 years and older) is projected to almost double from $14 \%$ to $20 \%$ of the total US population (i.e., one in every five persons or 70 million people) (3). Providing high level care for an increasing number of older breast cancer patients is of critical importance and will require health care providers and support staff who are trained in evaluating and treating this patient population (3).

The treatment of breast cancer combines surgery, systemic therapy, and radiotherapy.

The most common regimen for the complementary radiotherapy treatment of breast cancer after conservative $(6,7)$ or radical surgery has been the conventional fractionation, consisting of 45-50 Gy in 25 fractions of 1.8 or 2 Gy/day, 5 days a week, for 33 days $(8,9)$. Studies show that the incidence of local recurrence is significantly lower in the group of patients treated with radiotherapy, about an $8-10 \%$ with respect to the non-irradiated ones in which the local recurrence reached $25-35 \%$, independently of other associated factors $(9,10)$.

Elderly patients are frequently associated with concomitant diseases, cognitive impairment, socio-familial problems, or functional limitation (10). Patients often live far from the hospital and have difficulty in attending the radiotherapy sessions. Therefore, according to established practice it is not always feasible for elderly patients to come for a conventional daily radiotherapy treatment (11). This may lead to individuals avoiding complementary radiotherapy treatment after conservative surgery, or unnecessary abuse of radical mastectomy at the initial stages, which results in an under treatment or over treatment in a large number of cases. Many studies showed a clear under treatment in older patients due to difficulty in access and local recurrence $(12,13)$. Thus, the International Society for Geriatric Oncology guidelines strongly recommend the use of post mastectomy radiotherapy in the same conditions just as in younger population (14). Therefore, it is advisable to plan a more convenient treatment regimen for the elderly patients while a similar local control result can be achieved. Aside improvement in the quality of life, short treatments can also relieve the pressure on medical resources and the problem of access to radiotherapy (15).

One of the current challenges is the definition of irradiation schedules that are more comfortable for patients and easier to integrate with systemic therapies. In any case, there is, no clear consensus on what should be used on a regular basis $(16,17)$. This study therefore aimed to assess for a short radiotherapy regime with similar toxicity and outcome as the conventional radiotherapy in elderly breast cancer patients in the Usmanu Danfodiyo University, Sokoto, Nigeria. This if found, will increase access to radiotherapy facilities, and reduce logistics impediment in an environment with gross deficiency of radiotherapy facilities.

We present the following article in accordance with the STROBE reporting checklist (available at http://dx.doi. org/10.21037/tcr-20-1335).

\section{Methods}

This descriptive retrospective study used data from the records of all the elderly breast cancer patients who had mastectomy and were treated with hypofractionated radiotherapy in the Department of Radiotherapy and Oncology, Usmanu Danfodiyo University Teaching Hospital (UDUTH), Sokoto, Nigeria from January 2015 to February 2019. Patient's demographics, disease site(s), disease stage, histological characteristics, treatment toxicities and outcome data during 2-year follow ups were also retrieved. Patients with incomplete information in their records were dropped out of the study.

Radiotherapy treatment was delivered at a dose of 45 Gy in 18 daily fractions over 3.5 weeks using $6 \mathrm{MV}$ photons. Radiobiologically, assuming $\alpha / \beta$ ratio of 3.5 for breast cancer, our total dose equivalent in 2 Gy (EQD2) fraction is 49.09 Gy ( $\approx 50 \mathrm{~Gy})$.

The treatment was delivered to the chest wall by two opposed tangential fields. Supraclavicular area and axilla were treated with a direct field using same fractionation schedule. Several weekly clinical consultation and assessment reports during radiotherapy were retrieved from 
Table 1 Histological characteristics of the breast cancer cases

\begin{tabular}{lcc}
\hline Parameter & $\begin{array}{c}\text { No. of } \\
\text { patients }\end{array}$ & $\begin{array}{c}\text { Percentage } \\
(\%)\end{array}$ \\
\hline Stage & 27 & 32.5 \\
II & 56 & 67.5 \\
III & & \\
Hormone receptor status (HR) & 68 & 81.9 \\
HR positive (ER+ and/or PR+) & 10 & 12.0 \\
HR negative & 4 & 4.8 \\
HER2neu overexpression & 1 & 1.2 \\
Triple negative & 4 & 4.8 \\
Not specified & & \\
SBR grading & 9 & 10.8 \\
I & 53 & 63.9 \\
II & 21 & 25.3 \\
III & & \\
\hline
\end{tabular}

$\mathrm{HR}$, hormonal receptor, ER, estrogen receptor; PgR, progesterone receptor; SBR, Scarff-Bloom-Richardson grading.

the patients' case notes. Radiotherapy treatment toxicities were evaluated according to the Common Terminology Criteria for Adverse Effect (CTCAE) scale. After treatment, the patients' 3-monthly follow-up records for the first year, and 6-monthly follow-up records for the second year were evaluated. Treatment outcome and toxicities were sorted out and documented.

The study was conducted in accordance with the Declaration of Helsinki (as revised in 2013). Institutional ethical clearance was sought from the Ethical Committee of UDUTH, Sokoto, Nigeria (UDUTH/HREC/2020/No. 975). Permission to conduct the study was obtained from the management of the hospital. Individual consent for this retrospective analysis was waived due to the retrospective nature of the study.

\section{Statistical analysis}

Data were analyzed using the IBM Statistical Package for Social Sciences (SPSS) version 25 software (SPSS, IBM Corp, Armonk, NY, USA). Descriptive methods were used to present the patients' demographic data and disease characteristics using charts and frequency distribution tables. The disease free survival was calculated from the date of first presentation in the clinic with histological diagnosis of breast cancer to the first clinical evidence of cancer recurrence or death. The 2-year overall survival was calculated as those that were alive at 2-year follow up for those that came for the follow up or those we called and we got confirmation that they were alive at 2 years. Follow up beyond 2 years was very poor with up to $54 \%$ of the patients defaulting, hence the choice of 2-year-survival as end point in this study. Survival rates were calculated using Kaplan-Meier estimates.

\section{Results}

\section{Patient's characteristics}

Eighty-three elderly breast cancer cases that fulfilled the inclusion criteria were recruited into the study. The ages of the patients ranged from $60-80$ years with a mean age of $66.08 \pm 4.8$ years. All the patients had mastectomy. Fifty-eight $(69.9 \%)$ of the 83 patients had the disease on the right breast while $25(30.1 \%)$ had it on the left. The comorbidities observed among the patients were hypertensive heart diseases 18 (21.7\%), diabetes mellitus 9 (10.8\%) and osteoarticular diseases 38 (45.8\%).

The histological characteristics of the breast cancer cases are as presented in Table 1. The entire population of patients presented at either stage II or III with 27 (32.5\%) and $56(67.5 \%)$ patients respectively. Only 4 (4.8\%) out of all the patients had Her2neu over expression. One (1.2\%) presented with triple negative (ER-ve, PR-ve and Her2neuve) disease. Four (4.8\%) of the patients were referred to the center without immunohistochemistry report. Most patients $67(80.7 \%)$ had invasive ductal carcinoma (IDC), 7 (8.4\%) had invasive lobular carcinoma (ILC) and 9 (10.8\%) had other histologic types.

\section{Treatment modalities}

All the patients enrolled in this study had mastectomy as a form of surgery. Thirty-two (38.6\%) of them had upfront mastectomy, while $51(61.4 \%)$ had neoadjuvant chemotherapy before surgery. Among the 51 (61.4\%) patients that had neoadjuvant chemotherapy, 34 (66.7\%) received TAC (taxane, adriamycin and cyclophosphamide) three courses, followed by adjuvant chemotherapy with another 3 courses of the same regime. Nine (17.6\%) AC-T (adriamycin, cyclophosphamide, taxanes) protocol, 7 (13.7\%) FAC (5-flouracil, adriamycin cyclophosphamide) and $1(2.0 \%)$ EC (epirubicin, cyclophosphamide). Among those on hormone therapy 70 (84.3\%); 41 (58.6\%) were on 
aromatase inhibitor, $24(34.3 \%)$ on tamoxifen and 5 (7.1\%) were not any on any hormone therapy. Table 2 shows the radiotherapy modalities used among the study population. Lymph nodes irradiation was done in 71 patients (85.5\%); $40(56.3 \%)$ to the axilla, $31(43.7 \%)$ to the supraclavicular region.

\section{Toxicities}

Table 3 shows the toxicity rates during treatment and follow up. Skin erythema was observed in almost all the patients $78(94 \%)$ that received radiotherapy. Half of the patients $42(50.6 \%)$ had dry skin desquamation but no wet desquamation was recorded. Grade 1 nausea and vomiting was recorded in 37 (44.6\%) patients, while only 16 (19.2\%) had grade 2 nausea and vomiting.

\section{Disease recurrence}

After a median follow-up of 24 months duration, 3 local recurrences occurred; 2 (2.4\%) patients developed chest wall recurrence and 1 patient $(1.2 \%)$ had axillary nodal recurrence. The 2-year overall local recurrence rate was $3.6 \%$. Only 1 (1.2\%) patient had lung metastasis. Two-year distant metastasis rate was $1.2 \%$.

\section{Survival and death rates}

The 2-year overall survival pattern of the patients is

Table 2 Radiotherapy modalities used

\begin{tabular}{lcc}
\hline Radiotherapy site & $\begin{array}{c}\text { Number of } \\
\text { patients }\end{array}$ & $\begin{array}{c}\text { Percentage } \\
(\%)\end{array}$ \\
\hline Chest wall & 83 & 100 \\
LNRT ( $=71)$ & & \\
$\quad$ Axillary nodes & 40 & 56.3 \\
$\quad$ Supraclavicular nodes & 31 & 43.7 \\
\hline
\end{tabular}

presented in Figure 1. Eighty (96.4\%) were disease free at 24 months. Three deaths were recorded. The mean survival was 102.7 weeks (23.6 months) (95\% CI, 101-104) and the overall survival rate was $90.0 \%$.

\section{Discussion}

Elderly breast cancer patients experienced difficulties in accessing radiotherapy due to logistic and concomitant comorbidities. This problem is more grievous in Africa especially Nigeria where there are few radiotherapy machines, with 1 machine per 19,000,000 population as against the International Atomic Energy Agency recommendation of 1 mega voltage machine per 250,000 population (18-21).

The mean age of the study population was $66.08 \pm 4.8$, with an age range of $60-80$ years. A study conducted in France reported a similar finding with a mean age of 69 years and an age range of 53-86 years. Also a study in Genoa, Italy reported a median age of 81 years with a somewhat similar age range of 59-91 years $(22,23)$. A study conducted in North America by Khan et al., also reported a median age of 54 years (24).

A 10-year multi-center study in Nigeria reported that $62 \%$ of patients treated with post mastectomy radiotherapy were treated with various hypofractionation regimens in other to improve access and get more patients treated with radiotherapy. The most common hypofractionation regimen practiced in the country was 45 Gy in 18 fractions over 3.5 weeks (21).

Other hypofractionation schedules used in the country includes; 45 Gy in 15 fractions and 50 Gy in 20 fractions. The study also showed that most $(61.8 \%)$ of breast cancer patients in Nigeria were irradiated using hypofractionation schedule. Out of the 8 radiotherapy centers in the country, Lagos University Teaching Hospital in South western Nigeria and University of Nigeria Teaching Hospital Enugu, South eastern Nigeria practiced hypofractionation (45 Gy in 18 fractions) in all the patient treated within the

Table 3 Shows toxicities recorded according to Common Terminology Criteria for Solid Tumor

\begin{tabular}{lcccc}
\hline \multirow{2}{*}{ Reaction } & \multicolumn{3}{c}{ Grade } & 3 \\
\cline { 2 - 4 } & 1 & 2 & - & 4 \\
\hline Skin & $78(94 \%)$ & $42(50.6 \%)$ & - & - \\
Nausea/vomiting & $37(44.6 \%)$ & $16(19.2 \%)$ & - \\
\hline
\end{tabular}




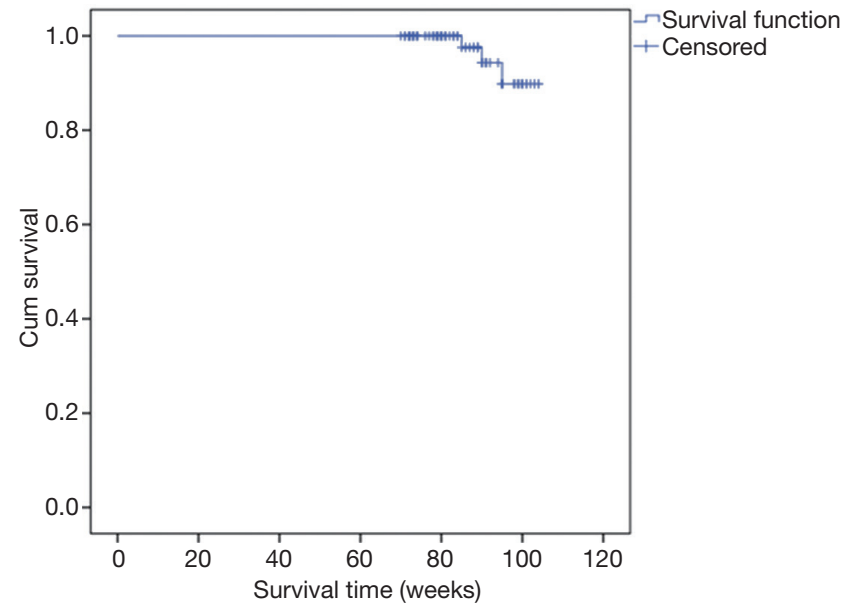

Figure 1 Overall survival of 83 breast cancer patients calculated by Kaplan-Meier estimates.

study period (21). In EKO hospital, a private radiotherapy center also in Lagos treated $13.5 \%$ of their patients using hypofractionation (50 Gy in 20 fractions). University College Hospital Ibadan in South Western Nigeria, treated $82.6 \%$ of their patients using same hypofractionation schedule. Other centers in the country used conventional fractionation in majority if not all of their patients: Ahmadu Bello University Teaching Hospital in North western Nigeria 98.3\%, National hospital Abuja 98.7\%, Usmanu Danfodiyo University Teaching Hospital Sokoto 99.6\% and all the patients treated in University of Benin Teaching Hospital Benin, South-South, Nigeria (21). In Nigeria, there is presently no national consensus guideline adopted for radiotherapy treatment. However, the Association of Radiation and Clinical Oncologist of Nigeria are currently working on one. The practice of radiotherapy fractionation described above cut across both the young and elderly breast cancer patients with no special consideration given to the elderly because of their peculiarity (21). Canadian trial used 42.5 Gy in 16 fractions over 22 days with favorable outcome (25). The START A (Standardization of Breast Radiotherapy) from the UK trial has shown that $41.6 \mathrm{~Gy} / 13$ fractions or $39 \mathrm{~Gy} / 13$ fractions are similar to the control regimen of $50 \mathrm{~Gy} / 25$ fractions in terms of local-regional tumor control and late normal tissue effects, a result consistent with the results of START trial $B$ which has shown that a radiation schedule of $40 \mathrm{~Gy} / 15$ fractions offers equivalent results to the standard schedule of 50 Gy/25 fractions (25-28).

To further simplify treatment in elderly breast cancer patients, a similar schedule of 42 or 45 Gy in 14 or 15 fractions over 4-5 weeks both after breast conserving surgery and mastectomy was used and presently, French guideline adopted the regimen for post mastectomy women (29). In Thailand Asia, 42.5 Gy in 16 fractions over 22 days was used with favorable outcome (30).

In this study, tolerance to hypofractionated schedule was good as most of the toxicities recorded were either grade 1 or grade 2 and therefore acceptable. Skin erythema was recorded in almost all the patient 78 (94\%). Half of the patients $42(50.6 \%)$ had dry skin desquamation but no wet desquamation was recorded. This is in consonant with TomoBreast trial comparing conventional versus hypofractionated radiotherapy which reported a reduction in Skin toxicity of grade $\geq 1$ at 2 years of $60 \%$ in conventional vs. $30 \%$ in in hypofractionated arm (31). Another long term study conducted in 18 centers in United Kingdom called Fast trial assessed breast changes comparing conventional radiotherapy to two shorter radiotherapy regimens (30 Gy in 5 weekly fractions and 28.5 Gy in 5 weekly fractions). The result of the study indicated that 1 weekly hypofractionation had similar tissue effect compared with conventional radiotherapy at 2 and 10 years follow up (32). Grade 1 nausea and vomiting was recorded in 37 (44.6\%) patients, while only $16(19.2 \%)$ had grade 2 nausea and vomiting. This nausea and vomiting was probably from the medications they were on as in most of them the complaint started before radiotherapy. Previous literature checks did not report any case of nausea and vomiting as side effect in their studies.

Cardiac and pulmonary toxicities were not recorded in any patient that received the treatment. This is in line with what Doré et al. reported in Europe in a study of 205 elderly breast cancer patients with no presentation of pulmonary or cardiac toxicities (23). TomoBreast trial also aligned with our findings where they reported that heart function showed no significant difference for left ventricular ejection fraction at 2 years, (Conventional $4.8 \%$ vs. Tomotherapy $4.6 \%)$. Pulmonary function tests at 2 years showed grade $\geq 1$ decline of FEV1 in $21 \%$ of conventional arm, vs. $15 \%$ of hypofractionated arm (30). Contrary to our finding, START trials reported up to $1.4 \%$ symptomatic pulmonary fibrosis, $2 \%$ ischemic heart disease and $1.3-1.5 \%$ rib fracture (26-28). However, we intend to continue following up the patient for any pulmonary, cardiac or other complications that may arise. Two patients presented to the clinic with lymphedema even before radiotherapy and hence, not included among 
the toxicities recorded in this study.

Lymph nodes irradiation was done in 71 patients $(85.5 \%)$; $40(56.3 \%)$ to the axilla, $31(43.7 \%)$ to the supraclavicular region without any significant increase in toxicities. Doré et al. in their study reported lymph nodes irradiation in 4 , 11 and 31 percent for axillary, internal mammary chain and supraclavicular region respectively without any increase in toxicities (23). TomoBreast trial also treated lymph nodes basins in those patients with node positive disease with no any increase in toxicities (31). Earlier studies like START A, B and Canadian studies were silent on lymph node irradiation (25-27).

Guenzi et al., in a study on hypofractionation from Kawasaki Medical School in Japan, concluded that shorter fractionation of radiotherapy following Breast conserving surgery has acceptable acute morbidity and can obtain a reasonably good cosmetic outcome (22). Other studies, three of which were clinical trials reported acceptable side effects in both breast conserving surgery and mastectomy with hypofractionation schedules $(22,25,26)$.

Contrary to our findings, Stoll et al. from Melbourne reported an unacceptable neuropathy rate of $73 \%$ in 1966 using $4 \mathrm{MeV}$ photon to a total of $63 \mathrm{~Gy}$ (33). A study in Sweden among breast cancer patients treated with CO60 machine to a total dose of 44 Gy in 11 fractions also reported a poor result with a large number presenting with fibrosis and neuropathy similar to the earlier study. Although, both studies used high doses of 114 Gy and 76 Gy using equivalent total dose of 2 Gy (EQD2) for Australian and Swedish study respectively (34).

After a median follow-up period of 24 months duration, 3 local recurrences occurred; 2 (2.4\%) patients developed chest wall recurrence and1patient $(1.2 \%)$ had axillary nodal recurrence. The 2-year overall local recurrence rate was $3.6 \%$. Only $1(1.2 \%)$ patient had lung metastasis (2-year distant recurrence rate was $1.2 \%)$. Previous studies from START A, START B and Cutuli et al. reported similar good outcomes with local recurrence rates of $3.2 \%, 2 \%$ and $3.7 \%$ respectively $(5,25,27)$. A study in Thailand with a follow-up period of 39 months also reported a good local control rate of $86 \%$ in elderly breast cancer women that were treated with hypofractionation regime (30). Lee et al. also reported in their study that elderly women treated with PMRT had significantly lower LRR compared with women treated without PMRT ( $16 \%$ vs. $28 \%, P=0.03$ ) (35).

The mean survival in this study was 102.7 weeks (23.6 months) (95\% CI, 101-104) and the overall 2-year survival rates was $90.0 \%$. At 24 months follow up, 3 deaths were recorded. Dore et al. and Cutuli et al. in a study with a long follow up of $\geq 5$ years reported a similar good survival with death rates of $37 \%$ and $33 \%$ respectively due to breast cancer $(5,23)$. Lee et al. also reported a good survival outcome in elderly women that received post mastectomy radiotherapy (35).

\section{Conclusions}

This study shows that hypofractionated radiotherapy is well tolerated in elderly breast cancer patients with a favorable outcome. This therefore seems to be an alternative to the long conventional fractionation schedules in breast cancer women.

\section{Acknowledgments}

Adamu Abdullahi contributed in editing the English language used.

Funding: None.

\section{Footnote}

Provenance and Peer Review: This article was commissioned by the Guest Editor (Vincent Vinh-Hung and Nam P. Nguyen) for the series "Radiotherapy for breast cancer in advanced age" published in the Translational Cancer Research. The article was sent for external peer review organized by the Guest Editor and the editorial office.

Reporting Checklist: The authors present the study in accordance with the STROBE reporting checklist. Available at http://dx.doi.org/10.21037/tcr-20-1335

Data Sharing Statement: Available at http://dx.doi. org/10.21037/tcr-20-1335

Conflicts of Interest: All authors have completed the ICMJE uniform disclosure form (available at http://dx.doi. org/10.21037/tcr-20-1335). The authors have no conflicts of interest to declare.

Ethical Statement: The authors are accountable for all aspects of the work in ensuring that questions related to the accuracy or integrity of any part of the work are appropriately investigated and resolved. The study was conducted in accordance with the Declaration of Helsinki (as revised in 2013). Institutional ethical clearance was sought 
from the Ethical Committee of UDUTH, Sokoto, Nigeria (UDUTH/HREC/2020/No. 975). Permission to conduct the study was obtained from the management of the hospital. Individual consent for this retrospective analysis was waived due to the retrospective nature of the study.

Open Access Statement: This is an Open Access article distributed in accordance with the Creative Commons Attribution-NonCommercial-NoDerivs 4.0 International License (CC BY-NC-ND 4.0), which permits the noncommercial replication and distribution of the article with the strict proviso that no changes or edits are made and the original work is properly cited (including links to both the formal publication through the relevant DOI and the license). See: https://creativecommons.org/licenses/by-nc-nd/4.0/.

\section{References}

1. Omran AR. The epidemiologic transition. A theory of the epidemiology of population change. Milbank Mem Fund Q 1971;49:509-38

2. Ferley J, Colombet M, Soerjomataram I, et al. Global and Regional Estimates of the incidence and Mortality for 38 Cancers: GLOBOCAN 2018. Lyon: International Agency for Research on Cancer/World Health Organisation; 2018. Available online: www.ncbi.nlm.nih.gov

3. Jemal A, Murray T, Ward E, et al. Cancer statistics, 2005. CA Cancer J Clin 2005;55:10-30.

4. Albrand G, Terret C. Early breast cancer in the elderly: assessment and management considerations. Drugs Aging 2008;25:35-45.

5. Cutuli B, De Lafontan B, Vitali E, et al. Breast conserving treatment (BCT) for stage I-II breast cancer in elderly women: analysis of 927 cases. Crit Rev Oncol Hematol 2009;71:79-88.

6. Algara M, Salinas J. Radiation therapy in breast cancer; Standard treatment and controversies, in Controversies in Radiotherapy for Breast Cancer, GlaxoSmithKline, Madrid, Spain, 2010:67-72.

7. Høst H, BrennhovdI O, Loeb M. Postoperative radiotherapy in breast cancer: Long-term results from the Oslo study. Int J Radiat Oncol Biol Phys 1986;12:727-32.

8. Fisher B, Redmond C. Lumpectomy for breast cancer: an update of the NSABP experience. National Surgical Adjuvant Breast and Bowel Project, J Natl Cancer Inst Monogr 1992;11:7-13.

9. Fisher B, Anderson S, Redmond CK, et al. Reanalysis and results after 12 years of follow-up in a randomized clinical trial comparing total mastectomy with lumpectomy with or without irradiation in the treatment of breast cancer. $\mathrm{N}$ Engl J Med 1995;333:1456-61.

10. Donato V, Valeriani M, Zurlo A. Short course radiation therapy for elderly cancer patients. Evidences from the literature review. Crit Rev Oncol Hematol 2003;45:305-11.

11. Punglia RS, Weeks JC, Neville BA, et al. Effect of distance to radiation treatment facility on use of radiation therapy after mastectomy in elderly women. Int J Radiat Oncol Biol Phys 2006;66:56-63.

12. Bouchardy C, Rapititi E, Florette G, et al. Under treatment strongly decrease prognosis of breast cancer in elderly women. J Clin Oncol 2003;21:3580-7.

13. Smith BD, Haffty BG, Huiria A, et al. Post mastectomy radiation and survival in older women with breast cancer. J Clin Oncol 2006;24:4901-7.

14. Biganzoli L, Wildiers H, Oakman E, et al. Management of elderly patient with breast cancer: updated recommendations of the International Society of Geriatric Oncology (SIOG) and European Society of Cancer Specialities (EUSOMA). Lancet Oncol 2012;13:e148-60.

15. Whelan TJ, Levine M, Julian J, et al. The effects of radiation therapy on quality of life of women with breast carcinoma: results of a randomized trial. Cancer 2000;88:2260-6.

16. Whelan T, Marcellus D, Clark R, et al. Adjuvant radiotherapy for early breast cancer: Patterns of practice in Ontario. CMAJ 1993;149:1273-7.

17. Mikeljevic JS, Haward R, Johnston C, et al. Trends in postoperative radiotherapy delay and the effect on survival in breast cancer patients treated with conservation surgery. Br J Cancer 2004;90:1343-8.

18. Dragun AE, Huang B, Tucker TC, et al. Disparities in the application of adjuvant radiotherapy after breast conserving surgery for early stage breast cancer: impact on overall survival. Cancer 2011;117:2590-8.

19. International Atomic Energy Agency. Setting up a Radiotherapy Programme: Clinical, Medical Physics, Radiation Protection and Safety Aspects. Vienna: International Atomic Energy Agency; 2008:6-45. Available online: https//www.pub.iaea.org

20. Ajogwu G. Nigeria Still Lacks Functioning Radiotherapy Machines@56. Daily Times [Internet]. 2016 [cited 25 August 2017]. Available online: http://dailytimes.ng/ nigeria-still-lacksfunctioning-radiotherapy-machines-56/

21. Omolola S, Malami AU, Alani JM, et al. Techniques and Practice of Post Mastectomy Radiotherapy in Nigeria: A Multi-Centre Study. Int J Oncol Cancer Res 2018;3:41-8. 
22. Guenzi M, Vagge S, Che N, et al. A biologically competitive 21 days hypofractionation scheme with weekly concomitant boost in breast cancer radiotherapy feasibility acute sub-acute and short term late effects. Radiation Oncology 2010;5:111.

23. Doré M, Cutuli B, Cellier P, et al. Hypofractionated irradiation in elderly patients with breast cancer after breast conserving surgery and mastectomy : Analysis of 205 cases. Radiat Oncol 2015;10:161.

24. Khan AJ, Poppe MM, Goyal S, et al. Hypofractionated Postmastectomy Radiation Therapy Is Safe and Effective: First Results From a Prospective Phase II Trial. J Clin Oncol 2017;35:2037-43.

25. Haviland JS, Owen JR, Dewar JA, et al. The UK standardization of Breast Radiotherapy (START) Trial of Radiotherapy hypofractionation for treatment of early breast cancer: 10 year follow up results of two randomized controlled trials. Lancet Oncol 2013;14:1086-94.

26. Whelan TJ, Pignol JP, Levine NM, et al. Long term result of hypofractionated radiotherapy for breast cancer. N Engl J Med 2010;362:513-20.

27. Bentzen SM, Agrawal RK, Aird EG, et al. The UK Standardisation of Breast Radiotherapy (START) Trial A of radiotherapy hypofractionation for treatment of early breast cancer: a randomised trial. Lancet Oncol 2008;9:331-41.

28. Bentzen SM, Agrawal RK, Aird EG, et al. The UK Standardisation of Breast Radiotherapy (START) Trial B of radiotherapy hypofractionation for treatment of early breast cancer: a randomised trial. Lancet 2008;371:1098-107.

29. Belkacemi Y, Fourquet A, Cutuli B, et al. French guidelines for infiltrating breast cancer. In: Cancer du sein infiltrant non metastatique-Questions d'actualities. INCa;2012. Available online: http;//www.e-cancer.fr/soins/ recommandations/cancers-du-sein. Accessed 10/10/2019.

30. Pinitpacharalert A, Chitapanarux I, Euathrongchit J, et al. A retrospective study comparing hypofractionated radiotherapy and conventional radiotherapy in post mastectomy breast cancer. J Med Assoc Thai 2011;94:94-102.

31. Van Parijs H, Miedema G, Vinh-Hung V, et al. Short course radiotherapy with simultaneous integrated boost for stage I-II breast cancer, early toxicities of a randomized clinical trial. Radiat Oncol 2012;7:80.

32. Brunt AM, Haviland JS, Sydenham M, et al. Ten-Year Results of FAST: A Randomized Controlled Trial of 5-Fraction Whole-Breast Radiotherapy for Early Breast Cancer. J Clin Oncol 2020;38:3261-72.

33. Stoll BA, Andrews JT. Radiation-induced peripheral neuropathy. BMJ 1966;1:834-7.

34. Johansson S, Svensson H, Denekamp J. Timescale of evolution of late radiation injury after postoperative radiotherapy of breast cancer patients. Int J Radiat Oncol Biol Phys 2000;48:745-50.

35. Lee JC, Truong PT, Speers HA, et al. Postmastectomy radiotherapy reduces locoregional recurrence in elderly women with high-risk breast cancer. Clin Oncol (R Coll Radiol) 2005;17:623-9.
Cite this article as: Aliyu UM, Kehinde A. Tolerance and outcome of hypofractionated post-mastectomy radiotherapy among elderly breast cancer patients in a specialized center in Nigeria. Transl Cancer Res 2020;9(11):6833-6840. doi: 10.21037/ tcr-20-1335 